(c) 2007 International Press

Adv. Theor. Math. Phys. 11 (2007) 175-197

\title{
Local mirror symmetry of curves: Yukawa couplings and genus 1
}

\author{
Brian Forbes $^{1}$ and Masao Jinzenji ${ }^{2}$ \\ ${ }^{1}$ Research Institute for Mathematical Sciences, Kyoto University, \\ Kyoto 606-8502, Japan \\ brian@kurims.kyoto-u.ac.jp \\ ${ }^{2}$ Division of Mathematics, Graduate School of Science, Hokkaido \\ University, Sapporo 060-0810, Japan \\ jin@math.sci.hokudai.ac.jp
}

\begin{abstract}
We continue our study of equivariant local mirror symmetry of curves, i.e., mirror symmetry for $X_{k}=\mathcal{O}(k) \oplus \mathcal{O}(-2-k) \rightarrow \mathbb{P}^{1}$ with torus action $\left(\lambda_{1}, \lambda_{2}\right)$ on the bundle. For the antidiagonal action $\lambda_{1}=-\lambda_{2}$, we find closed formulas for the mirror map, a rational $B$ model Yukawa coupling and consequently Picard-Fuchs equations for all $k$. Moreover, we give a simple closed form for the $B$ model genus 1 Gromov-Witten potential. For the diagonal action $\lambda_{1}=\lambda_{2}$, we argue that the mirror symmetry computation is equivalent to that of the projective bundle $\mathbb{P}(\mathcal{O} \oplus \mathcal{O}(k) \oplus \mathcal{O}(-2-k)) \rightarrow \mathbb{P}^{1}$. Finally, we outline the computation of equivariant Gromov-Witten invariants for $A_{n}$ singularities and toric tree examples via mirror symmetry.
\end{abstract}

e-print archive: http://lanl.arXiv.org/abs/math/0609016 


\section{Introduction}

Mirror symmetry has for some time now provided a convenient shortcut in the computation of Gromov-Witten invariants of toric varieties. Although mirror symmetry for compact Calabi-Yau toric varieties historically appeared first, it has since been realized that the noncompact (or local) CY toric variety setting enjoys many simplifications not present in the compact case.

However, as it turns out, there is a price to be paid for these simplifications: when working with noncompact Calabi-Yaus, one loses the nice structure theorems regarding periods of the mirror Calabi-Yau manifold. For example, on compact three-folds, the constant term of the prepotential (generating function of genus 0 Gromov-Witten invariants) is known to be given by the triple intersection number of curve classes in the space. However, for noncompact spaces, we have no triple intersection number, and often the natural choice for this number (from the perspective of mirror symmetry) turns out to be fractional.

More along the lines of the present paper, we are unable to use mirror symmetry for any Calabi-Yau three-fold $X$ such that $b_{4}(X)=0$. This is because the periods mirror to the four cycles of $X$ are used to compute the prepotential. As an even more serious problem, consider the case of 'local mirror symmetry of $\mathbb{P}^{1}$ ', by which we mean mirror symmetry for $X_{k}=$ $\mathcal{O}(k) \oplus \mathcal{O}(-2-k) \rightarrow \mathbb{P}^{1}$. The reason for this terminology is that if $M$ is a Calabi-Yau three-fold containing an imbedded $\mathbb{P}^{1}, \mathbb{P}^{1} \hookrightarrow M$, then the local Calabi-Yau condition implies

$$
N_{\mathbb{P}^{1} / X} \cong \mathcal{O}(k) \oplus \mathcal{O}(-2-k)
$$

This space is actually greatly troubled, as the Gromov-Witten theory of $X_{k}$ for $k \geq 0$ is not even well-defined! This was shown in [4], where it was found that the Gromov-Witten invariants change drastically with different choices of equivariant weights $\left(\lambda_{1}, \lambda_{2}\right)$ acting on the bundle. As such, any mirror symmetry construction for $X_{k}$ will necessarily be one of equivariant mirror symmetry.

Thus, in this paper, we develop a version of mirror symmetry for $X_{k}$ with torus weights $(\lambda,-\lambda)$ acting on the bundle. This case is of special interest, as this is the Gromov-Witten theory computed by physicists [14]. We show that through a certain decomposition of the bundle, we are able to describe mirror symmetry at genus 0 (i.e., the mirror map and Yukawa coupling) via very simple rational functions. Moreover, this structure allows us to easily work out mirror symmetry at genus 1 . 
After this paper was completed, we were informed by the authors of [14] that they had obtained the same formula for the mirror map and genus 1 partition function in their paper. In contrast to our work here, the computations of [14] are ultimately from the topological vertex, or $A$ model, perspective, and their mirror map was found as a 'natural' variable for the problem, rather than a mirror map in the strict sense. Here, we will perform all calculations using only the techniques of mirror symmetry - and we do find the same mirror map can be used, though this mirror map actually belongs to a different space, as we will see.

The organization of this paper is as follows. Section 2 summarizes our main results. In Section 3, we review our previous results [9], and give a derivation of natural rational Yukawa couplings and genus 1 mirror symmetry on $X_{k}$ (with antidiagonal action $(\lambda,-\lambda)$ ). Finally, in Section 4 we apply equivariant mirror symmetry to two spaces which are not of the bundleover- $\mathbb{P}^{1}$ type.

\section{Overview}

We state our main results and methods in this section. Throughout, we take $X_{k}=\mathcal{O}(k) \oplus \mathcal{O}(-2-k) \rightarrow \mathbb{P}^{1}$ and equip the bundle with a torus action with weights $\left(\lambda_{1}, \lambda_{2}\right)$. Let $t_{k}$ to be the Kähler class of $\mathbb{P}^{1} \hookrightarrow X_{k}$.

\subsection{Curves}

We consider first the antidiagonal action $\lambda_{1}=-\lambda_{2}$ case. This is the computation which is of interest to physicists, and is the so-called equivariantly Calabi-Yau setting. We can exhibit the Calabi-Yau property by observing that the sum of the column vectors of the matrix of charge vectors for $X_{k}$ is zero when $\lambda_{1}=-\lambda_{2}$ :

$$
\left(\begin{array}{cccc}
1 & 1 & k & -2-k \\
0 & 0 & \lambda_{1} & \lambda_{2}
\end{array}\right)
$$

Mirror symmetry was of course first observed for Calabi-Yau manifolds, and many of the nice structures associated to quantum cohomology, etc. owe themselves to this property. As such, although equivariant GromovWitten invariants for $X_{k}$ have been to this point not well understood, one may hope that equivariantly Calabi-Yau spaces possess the same structure. We have found that in fact this is the case: 
Conjecture 1. For $X_{k}=\mathcal{O}(k) \oplus \mathcal{O}(-2-k) \rightarrow \mathbb{P}^{1}$ with antidiagonal torus action $(\lambda,-\lambda)$ on the bundle, the mirror map is given by

$$
q \frac{d t_{k}}{d q}=\frac{1+(-1)^{k+1}(k+1)^{2} q}{1+(-1)^{k+1} q}
$$

and the rational B model Yukawa coupling, by the formula

$$
Y_{q q q}^{k}=\frac{-1}{k(k+2)}\left(q \frac{d t_{k}}{d q}\right)^{2}
$$

In particular, this implies that we have the following Picard-Fuchs equation describing mirror symmetry for $X_{k}$ :

$$
\partial_{t_{1}}\left(\frac{1}{Y_{t_{1} t_{1} t_{1}}^{k}}\right) \partial_{t_{1}}^{2}=\theta^{2}\left(q \frac{d t_{k}}{d q}\right)^{-1} \theta
$$

As mentioned in the introduction, the mirror map above was found in [14] as a 'natural' variable for this calculation, and it was then speculated in [14] based on integrality properties that this formula might be interpreted as a mirror map. Here, we have found directly from mirror symmetry that this is indeed the mirror map, though technically, the mirror map of a different space (see Proposition 1 below).

We note in particular that this formula implies that the constant term of the $A$ model Yukawa coupling will be fractional:

$$
Y_{t_{k} t_{k} t_{k}}^{k}=\frac{\partial^{3} \mathcal{F}_{k}}{\partial t_{k}^{3}}=\left(q \frac{d t_{k}}{d q}\right)^{-3} Y_{q q q}^{k}=\frac{-1}{k(k+2)}+O\left(e^{t}\right) .
$$

where $\mathcal{F}_{k}$ is the prepotential for $X_{k}$. This is precisely the value that was predicted (through entirely different considerations) in [2]. As this constant has the interpretation of being the triple intersection number of $\mathbb{P}^{1} \hookrightarrow X_{k}$, we see the fractionality of intersection numbers which was observed previously in $[11,7,8]$. We note that this choice of triple intersection number is not the unique one that gives a rational Yukawa coupling; however, this choice gives the simplest form of the $B$ model Yukawa coupling and Picard-Fuchs equations, hence making it natural from the $B$ model perspective.

This same elementary structure is also present at genus 1:

Conjecture 2. The genus 1 Gromov-Witten potential of $X_{k}$ with the antidiagonal torus action is given in $B$ model variables by the closed formula

$$
\begin{aligned}
G_{k}= & \frac{11}{24} \log \left(1+(-1)^{k+1}(k+1)^{2} q\right)+\left(-\frac{5}{12}+\frac{(k+1)^{2}}{24}\right) \\
& \times \log \left(1+(-1)^{k+1} q\right)-\frac{1}{2} \log \left(q \frac{d t_{k}}{d q}\right)
\end{aligned}
$$


This formula was derived, in a slightly different form, in [14], by looking directly at the $A$ model calculation. Here, we have found this through mirror symmetric methods, by interpreting the singular points of the mirror map as the discriminant locus.

These conjectures were arrived at by use of the following proposition:

Proposition 1. The equivariant Gromov-Witten invariants of

$$
X_{k}=\mathcal{O}(k) \oplus \mathcal{O}(-2-k) \rightarrow \mathbb{P}^{1}
$$

with action $\left(\lambda_{1}, \lambda_{2}\right)$ on the bundle are the same as those of the total space

$$
X_{k}^{\prime}=\oplus_{1}^{k} \mathcal{O}(1) \bigoplus \oplus_{1}^{2+k} \mathcal{O}(-1) \rightarrow \mathbb{P}^{1}
$$

with $(\overbrace{\lambda_{1} \cdots \lambda_{1}}^{k} ; \overbrace{\lambda_{2} \cdots \lambda_{2}}^{2+k})$ acting on the bundle.

This 'factorization' of the bundle of $X_{k}$ into a sum of $\mathcal{O}(1)$ and $\mathcal{O}(-1)$ terms has the effect of dramatically simplifying the mirror map in the case of the antidiagonal action. We will see that this proposition is actually the natural generalization of the results found in [9]. Thus, the 'mirror map' we have found above for the antidiagonal torus action is actually the mirror map of $X_{k}^{\prime}$, rather than $X_{k}$.

To see why the above proposition is true, we have the following argument due to Iritani. Let $p$ be the Kähler class measuring the volume of the $\mathbb{P}^{1}$. Then we compare the equivariant Euler class of the bundle of $X_{k}$ :

$$
\begin{gathered}
e_{T}(\mathcal{O}(k) \oplus \mathcal{O}(-2-k))=\left(k p+\lambda_{1}\right)\left((-2-k) p+\lambda_{2}\right)= \\
\left(k \lambda_{2}-(2+k) \lambda_{1}\right) p+\lambda_{1} \lambda_{2}
\end{gathered}
$$

to that of $X_{k}^{\prime}$ :

$$
\begin{gathered}
e_{T}\left(\oplus_{1}^{k} \mathcal{O}(1) \bigoplus \oplus_{1}^{2+k} \mathcal{O}(-1)\right)=\left(p+\lambda_{1}\right)^{k}\left(-p+\lambda_{2}\right)^{2+k}= \\
\left(\left(k \lambda_{2}-(2+k) \lambda_{1}\right) p+\lambda_{1} \lambda_{2}\right) \lambda_{1}^{k-1} \lambda_{2}^{2+k-1}
\end{gathered}
$$

where we have imposed the cohomology relation $p^{2}=0$. Then we see that these are effectively the same (up to the multiplicative factor $\lambda_{1}^{k-1} \lambda_{2}^{2+k-1}$, which can be thought of as simply a product of trivial bundles). As the theorem of [5] uses only this equivariant Euler class as input, one concludes that the equivariant Gromov-Witten invariants of both spaces must be equal. 
Although we can prove the proposition this way, this equivalence was originally deduced through more geometric considerations, which are described in the body of the paper. Yet even with this proposition in hand, it is quite nontrivial that we find the remarkable structure listed above for the antidiagonal action, i.e., the simplified mirror map and, most dramatically, the existence of the rational Yukawa coupling.

The diagonal action $\lambda_{1}=\lambda_{2}$ unfortunately does not possess the nice structure observed above. This is not terribly surprising, as this case is manifestly not Calabi-Yau. Nonetheless, we find the following phenomenon:

Conjecture 3. The equivariant mirror symmetry computation on $X_{k}$ with the diagonal torus action is the same as that of $\mathbb{P}(\mathcal{O} \oplus \mathcal{O}(k) \oplus \mathcal{O}(-2-k))$. That is, the mirror maps and Gromov-Witten invariants are equal.

We can gain some understanding of how this comes about by examining the charge vectors of the projective bundle spaces:

$$
\mathbb{P}(\mathcal{O} \oplus \mathcal{O}(k) \oplus \mathcal{O}(-2-k)):\left(\begin{array}{ccccc}
1 & 1 & k & -2-k & 0 \\
0 & 0 & 1 & 1 & 1
\end{array}\right)
$$

By looking at this matrix and then back at (2.2), we see that the toric data of these two spaces is essentially equivalent, and since the $I$ functions are determined entirely from the above matrices, it is not too outlandish that we should find the same mirror maps and Gromov-Witten invariants between these examples.

\section{2 $A_{n}$ singularities and toric trees}

In [9], it was suggested that the Gromov-Witten invariants that physicists use are often actually equivariant Gromov-Witten invariants. Here, we realize this idea by computing the prepotential and genus 1 GromovWitten potential for $A_{n}$ singularities and for three-folds $X$ satisfying dim $H_{4}(X, \mathbb{Z})=0$.

In [8], we showed that one could compute the prepotential of a noncompact Calabi-Yau three-fold up to polynomial terms of degree 2 by using various compactifications. However, particularly in the no 4 cycle $\operatorname{dim} H_{4}(X, \mathbb{Z})=0$ case, this approach is not satisfactory, since we deliberately use a compactification known to reproduce the physically expected answer. Here, we will see that through the equivariant formalism, physical Gromov-Witten invariants appear naturally. 
We first consider the $A_{n}$ singularity. This geometry is described by the $n \times(n+2)$ matrix

$$
\left(\begin{array}{ccccccc}
1 & -2 & 1 & 0 & 0 & \cdots & 0 \\
0 & 1 & -2 & 1 & 0 & \cdots & 0 \\
\vdots & & & & & & \\
0 & 0 & 0 & \cdots & 1 & -2 & 1
\end{array}\right)
$$

This space has $n 2$ cycles arranged along a line corresponding to the Dynkin diagram of $A_{n}$; we label these sequentially by $C_{1} \cdots C_{n}$. Let $t_{1} \cdots t_{n}$ be the complexified Kähler classes corresponding to these curves. Then we work with the equivariant theory

$$
\left(\begin{array}{ccccccc}
0 & \lambda_{1} & \lambda_{2} & \cdots & \lambda_{n-1} & \lambda_{n} & 0 \\
1 & -2 & 1 & 0 & 0 & \cdots & 0 \\
0 & 1 & -2 & 1 & 0 & \cdots & 0 \\
\vdots & & & & & & \\
0 & 0 & 0 & \cdots & 1 & -2 & 1
\end{array}\right)
$$

The idea behind this choice is the same as that of the $\mathcal{O} \oplus \mathcal{O}(-2) \rightarrow \mathbb{P}^{1}$ case considered in [9]: each -2 entry corresponds to a noncompact divisor, so we 'compactify' these divisors by adding in an equivariant parameter for each. Then, we simply use the equivariant $I$ function to extract GromovWitten invariants via mirror symmetry. The result for the prepotential is the following:

$$
\mathcal{F}_{A_{n}}=\sum_{i=1}^{n} L i_{3}\left(e^{t_{i}}\right)+\sum_{i=1}^{n-1} L i_{3}\left(e^{t_{i}+t_{i+1}}\right)+\cdots+L i_{3}\left(e^{t_{1}+\cdots+t_{n}}\right) .
$$

Later in the paper, we use this instanton expansion, together with the discriminant locus computed from a $\mathbb{P}^{1}$ fibration over the $A_{2}$ singularity, to exhibit mirror symmetry at genus 1 on $A_{2}$.

Now let $X$ be any noncompact Calabi-Yau three-fold with no 4 cycles, such that $X$ is described by symplectic reduction via a matrix $M_{a b}$. As is well known, by making appropriate choices of curves $C_{1} \cdots C_{j}$ and divisors $D_{1} \cdots D_{k}$ in $X$, the entries of $M_{a b}$ give intersection numbers between curves and divisors. Suppose that $D_{1} \cdots D_{l}$ is a basis of noncompact divisors of $X$. Then as above, we consider the equivariant Gromov-Witten theory of 
$X$ with exactly one equivariant parameter inserted for each noncompact divisor:

$$
\left(\begin{array}{cccc}
\lambda_{1} \cdots & \lambda_{l} & 0 \cdots & 0 \\
m_{1,1} \cdots & m_{1, l} & m_{1, l+1} \cdots & m_{1, k} \\
\vdots & & & \\
m_{j, 1} \cdots & m_{j, l} & m_{j, l+1} \cdots & m_{j, k}
\end{array}\right)
$$

Again, only the equivariant $I$ function is required to compute GromovWitten invariants. The result for the prepotential is nearly the same as the above: we get one term in the prepotential for each curve in the geometry. However, there is one important difference: by tuning the equivariant parameters, we can arrange things so that the curves with normal bundle $\mathcal{O}(-1) \oplus$ $\mathcal{O}(-1)$ and those with normal bundle $\mathcal{O} \oplus \mathcal{O}(-2)$ have either the same relative sign, or the opposite relative sign. Moreover, the choice in which the curves have opposite relative sign corresponds to the physical 'anti-diagonal action' case, which is consistent with physically computed prepotentials. However, from the equivariant point of view, either sign convention is equally acceptable, as was suggested in [8].

As the prepotential cannot be written in a concise form, we will instead work out the explicit example of the trivalent $(-1,-1)$ curve in the text, computing the prepotential and subsequently exhibiting genus 1 mirror symmetry.

\section{Equivariant local mirror symmetry of curves}

\subsection{Review of previous results}

We begin with an overview of the findings of [9]. What was shown was essentially that we can use the equivariant version of the Givental $I$ function to compute equivariant Gromov-Witten invariants of $X_{k}$ with the general torus action $\left(\lambda_{1}, \lambda_{2}\right)$ on the bundle. For $X_{-1}$, the equivariant $I$ function reads

$$
I_{-1}^{\lambda}=e^{p \log q / \hbar} \sum_{d \geq 0} \frac{\prod_{i=1,2} \prod_{m=-d+1}^{0}\left(-p+m \hbar+\lambda_{i}\right)}{\prod_{m=1}^{d}(p+m \hbar)^{2}} q^{d}
$$

and on $X_{0}$,

$$
I_{0}^{\lambda}=e^{p \log q / \hbar} \sum_{d \geq 0} \frac{\prod_{m=-2 d+1}^{0}(-2 p+m \hbar+\lambda)}{\prod_{m=1}^{d}(p+m \hbar)^{2}} q^{d} .
$$


These $I$ functions are annihilated by the following two equivariant differential operators, respectively:

$$
\begin{aligned}
\mathcal{D}_{-1} & =\theta^{2}-q\left(\theta-\lambda_{1}\right)\left(\theta-\lambda_{2}\right), \\
\mathcal{D}_{0} & =\theta^{2}-q(2 \theta-\lambda)(2 \theta-\lambda+\hbar) .
\end{aligned}
$$

where $\theta=\hbar q d / d q$. As was shown in [9], these two $I$ functions agree up to the mirror map and equivariant mirror map if we take $\lambda_{1}=\lambda_{2}=\lambda$ in $I_{-1}^{\lambda}$. This means that the two equivariant differential equations $\mathcal{D}_{-1} f=0, \mathcal{D}_{0} f=0$ generate the same quantum cohomology ring when $\lambda_{1}=\lambda_{2}$.

We note one unusual feature of $I_{0}^{\lambda}$ which will be key to the derivations that follow. The issue is that the $I$ function is unable to detect the trivial $\mathbb{C}$ factor of $X_{0}=\mathcal{O} \oplus \mathcal{O}(-2) \rightarrow \mathbb{P}^{1}$. This implies two things. First, the $I$ function cannot be used to compute the minus sign on the instanton expansion of $X_{0}$ claimed by physicists for the antidiagonal action (e.g. [14]). Secondly, the point which is central to this paper: $I_{0}^{\lambda}$ is actually the equivariant $I$ function of the $A_{1}$ singularity. In other words, the equivalence between $I_{-1}^{\lambda_{1}=\lambda_{2}}$ and $I_{0}^{\lambda}$ in [9] is an equivalence of equivariant quantum cohomology rings between the three dimensional space $X_{0}$ and the two dimensional $A_{1}$ singularity. The natural generalization of this observation is the proposition of the previous section. We will see how this can be derived for $\mathcal{O}(1) \oplus \mathcal{O}(-3) \rightarrow \mathbb{P}^{1}$ with the antidiagonal action later in the paper.

As one final remark on the above, we compare the equivariant charge vectors of $X_{-1}$ and the $A_{1}$ singularity:

$$
\begin{aligned}
& X_{-1}:\left(\begin{array}{cccc}
1 & 1 & -1 & -1 \\
0 & 0 & \lambda & \lambda
\end{array}\right) \\
& A_{1}:\left(\begin{array}{ccc}
1 & 1 & -2 \\
0 & 0 & \lambda
\end{array}\right)
\end{aligned}
$$

From this vantage, the calculation on $X_{-1}$, while equivalent to that of $A_{1}$, is slightly simpler, because there is no mirror map. So, the dimension of the space has gone up, and the complexity of the mirror map has gone down. This is the first example of the 'factorization of the bundle' stated in Proposition 1.

Moving on, from [9] we have the equivariant $I$ function for $X_{k}$ for $k \geq 1$ :

$$
I_{k}^{\lambda}=e^{p \log q / \hbar} \sum_{d \geq 0} \frac{\prod_{m=(-2-k) d+1}^{0}\left((-2-k) p+m \hbar+\lambda_{2}\right)}{\prod_{m=1}^{d}(p+m \hbar)^{2} \prod_{m=1}^{k d}\left(k p+m \hbar+\lambda_{1}\right)} q^{d} .
$$


Then we proposed that the equivariant Gromov-Witten invariants of $X_{k}$ could be recovered by first expanding $I_{k}^{\lambda}$ about $\lambda_{1}=\infty$, then performing Birkhoff factorization of the result (to remove positive powers of $\hbar$ ), and finally by inverting the mirror map and equivariant mirror map of the Birkhoff factorized function (which we called the $J$ function).

We briefly recall the Birkhoff factorization procedure. Since the only examples we work with will be curves, we can give an especially simple formulation. Suppose we have an equivariant $I$ function representing some bundle over a curve, and that after expansion about the equivariant parameters $\lambda=\infty$, we obtain $I \in \mathbb{C}\left[\hbar, \hbar^{-1}\right]$. Since such a power series expansion strictly speaking does not make sense, we have to remove positive powers of $\hbar$ from the $I$ function before extracting mirror symmetry data. This is done by a theorem in [5]: there exist functions $c_{0}, c_{1}$ such that

$$
c_{0}(q, \hbar) I\left(q, \hbar, \hbar^{-1}\right)+c_{1}(q, \hbar) \hbar q \frac{d}{d q} I\left(q, \hbar, \hbar^{-1}\right)=J\left(q, \hbar^{-1}\right)
$$

and $J$ is independent of $\hbar$. We then obtain the mirror map, etc. by looking at the $\hbar^{-1}$ expansion of $J$.

Then the above process of equivariant mirror symmetry, given in slightly more detail, proceeds by performing the series expansion and Birkhoff factorization, from which we find

$$
J=1+\frac{t_{1}^{\lambda}(q) p+t_{2}^{\lambda}(q)}{\hbar}+\frac{W_{1}^{\lambda}(q) p+W_{2}^{\lambda}(q)}{\hbar^{2}}+\cdots
$$

Then by multiplying $J$ by $e^{-t_{2}^{\lambda}(q)}$ and inverting the mirror map $t_{1}^{\lambda}(q)$, we can read off the instanton information from the $W_{i}(q)$. It was then shown that if $\lambda_{1}=\lambda_{2}$, the resulting functions are independent of $k$, and for $\lambda_{1}=-\lambda_{2}$, physical Gromov-Witten invariants for $X_{k}$ could be computed.

Unfortunately, this method produces no closed formulas, and requires serious computer power even to obtain results up to degree 6 . Moreover, the resulting mirror maps have incredibly complicated formulas. For example, on the $X_{1}$ geometry with the diagonal torus action, the formula for the mirror map is given by

$$
\frac{d \log q}{d t}=\frac{3}{8}\left(1+\frac{\sin \left(5 / 3 \sin ^{-1}(\sqrt{108 q})\right)}{\sqrt{108 q}}\right) .
$$

These types of formulas have put the search for Yukawa couplings, as well as the genus 1 computation, out of reach. We were unable to even identify a discriminant for this case. 
These problems, and the desire to perform the $B$ model at genus 1 , led us to search more carefully for the meaning of mirror symmetry for these spaces. The first clue is given by a close look at $D_{n}$ singularities.

\subsection{Connection between $X_{1}$ and $D$ type singularities}

In the previous section, we have explored the direct approach to mirror symmetry for $X_{k}$ via $I$ functions, and have noted along the way that the $X_{0}$ calculation can be viewed as equivariant theory on the $A_{1}$ singularity. Next, we claim that the equivariant Gromov-Witten invariants of $X_{1}=\mathcal{O}(1) \oplus$ $\mathcal{O}(-3) \rightarrow \mathbb{P}^{1}$ are the same as the equivariant Gromov-Witten invariants of a certain partial resolution of the $\mathbb{C}^{2} / D_{n}$ singularity.

Recall that a simple singularity $\mathbb{C}^{2} / D_{n}, n \geq 4$, where $D_{n}$ is the $n$th dihedral group, can be realized as a hypersurface in $\mathbb{C}^{3}[10]$

$$
f=x_{1}^{2} x_{2}-x_{2}^{n-1}+x_{3}^{2}=0 .
$$

One can obtain a smooth variety in two ways. One way is complex deformation: take a basis $\left\{\mu_{1} \cdots \mu_{m}\right\}$ of the local algebra of the singular point

$$
H=\frac{\mathbb{C}\left[x_{1}, x_{2}, x_{3}\right]}{\left\langle\partial_{x_{1}} f, \partial_{x_{2}} f, \partial_{x_{3}} f\right\rangle}
$$

and deform $f$ as $f^{\lambda}=f+\sum_{j=1}^{m} \lambda_{j} \mu_{j}$. The second way is by blowing up the $n$ singular points; we end up with $n$ curves of self-intersection -2 , and moreover, there is a special 'central' curve which intersects three other curves exactly once. We call this the trivalent curve. Mirror symmetry for the $D_{n}$ singularity is then realized as the transformation between the blown up space and the complex deformed space.

We next recall the work of Cachazo et al. [6], where it was shown (in the so-called Laufer's example section, pp. 37-40) that a certain monodromic fibration of the $D_{n}$ singularity over the plane, where only the trivalent curve is blown up, is equivalent to the geometry $X_{1}=\mathcal{O}(1) \oplus \mathcal{O}(-3) \rightarrow \mathbb{P}^{1}$. Now, in our case, we cannot use this fact directly, because we are interested in the mirror symmetry computation. From the vantage of mirror symmetry, everything is much simpler if we stay in the realm of toric geometry. Nonetheless, there is reason to suspect that we can still find a relationship between $X_{1}$ and $D_{n}$ singularities at the toric level; see for example [12], where the connection is described as follows. If we let $X$ be a Calabi-Yau three-fold with imbedded curve $\mathbb{P}^{1} \hookrightarrow X$ such that $N_{\mathbb{P}^{1} / X} \cong \mathcal{O}(1) \oplus \mathcal{O}(-3)$, and we then shrink the $\mathbb{P}^{1}$ to a point $p$, then under certain conditions the 
singularity type of the generic hyperplane section through the point $p$ will be of type $D_{4}$.

Thus, we would like to consider the toric representation of the $D_{n}$ geometry in which only the trivalent curve is blown up. From [13], the Mori cone vector corresponding to this trivalent curve is given by

$$
\left(\begin{array}{lllll}
1 & 1 & 1 & -2 & -1
\end{array}\right),
$$

and therefore we end up with a geometry defined by single Kähler parameter. We call this the " $D_{1}$ singularity".

We now show that this is indeed the Mori cone generator of the trivalent curve. Consider for simplicity the blown up $D_{4}$ geometry, and let $C_{1} \cdots C_{4}$ be a basis of curve classes, where $C_{4}$ is the trivalent curve. We claim that the toric data defining the blow up of the $D_{4}$ singularity is given by the matrix

$$
\left(\begin{array}{l}
l_{1} \\
l_{2} \\
l_{3} \\
l_{4}
\end{array}\right)=\left(\begin{array}{cccccccc}
-2 & 0 & 0 & 1 & 0 & 1 & 0 & 0 \\
0 & -2 & 0 & 1 & 0 & 0 & 1 & 0 \\
0 & 0 & -2 & 1 & 0 & 0 & 0 & 1 \\
1 & 1 & 1 & -2 & -1 & 0 & 0 & 0
\end{array}\right)
$$

We can derive this matrix as follows. The rows of the matrix, as well as the first four columns, correspond to the curves $C_{1} \cdots C_{4}$, and entries of the matrix give intersection numbers between curves and divisors in the geometry. Hence the -2 entries are interpreted as the self-intersection numbers $C_{i}^{2}=-2$, and e.g., the meaning of the $(4,1)$ entry is $C_{1} \cdots C_{4}=1$, which is true because $C_{4}$ intersects $C_{1} \cdots C_{3}$ exactly once. Thus we see the necessity of the three 1's in the fourth row. Also, the last three columns represent the (noncompact) normal bundles to the curves $C_{1} \cdots C_{3}$ respectively, and since the intersection number of each curve with its normal bundle is +1 , we obtain the entries of these columns. Finally, in order to impose the CalabiYau condition on the space, we need to add the -1 in the fifth column (so $\left.\sum_{j} l_{4}^{j}=0\right)$. Hence we arrive at the claimed form of the Mori vector (3.30).

Since this is the matrix of intersection numbers of the space, we can represent blown up $D_{4}$ as a complex four dimensional space given by

$$
\frac{\left\{\left(z_{1} \cdots z_{8}\right) \in \mathbb{C}^{8}: \sum_{j=1}^{8} l_{i}^{j}\left|z_{j}\right|^{2}=r_{i}, i=1 \cdots 4\right\}}{\left(S_{1}\right)^{4}}
$$

where $r_{i}$ are real parameters and the action is given by

$$
S_{i}^{1}:\left(z_{1} \cdots z_{8}\right) \longrightarrow\left(e^{l_{i}^{1} \sqrt{-1} \theta_{1}} z_{1} \cdots e^{l_{i}^{8} \sqrt{-1} \theta_{8}} z_{8}\right)
$$


We now return to the discussion of the relation between the $D_{1}$ geometry and that of $X_{1}$. Notice that the charge vector of $X_{1}=\mathcal{O}(1) \oplus \mathcal{O}(-3) \rightarrow \mathbb{P}^{1}$ is given as:

$$
\left(\begin{array}{llll}
1 & 1 & 1 & -3
\end{array}\right) \text {. }
$$

If we stare at the vectors in equations (3.34), (3.30) and then look back at equation (3.22), it is not hard to imagine that the following two equivariant theories would give the same Gromov-Witten invariants:

$$
\begin{aligned}
& D_{1}:\left(\begin{array}{ccccc}
1 & 1 & 1 & -1 & -2 \\
0 & 0 & \lambda_{1} & \lambda_{2} & \lambda_{2}
\end{array}\right) \\
& X_{1}:\left(\begin{array}{cccc}
1 & 1 & 1 & -3 \\
0 & 0 & \lambda_{1} & \lambda_{2}
\end{array}\right)
\end{aligned}
$$

Indeed, direct computation verifies the equality of the Gromov-Witten invariants. We note that as in the $X_{-1} / A_{1}$ case, the dimension of the spaces is different. The charge vector of the $D_{1}$ geometry identifies the space as $\mathcal{O}(1) \oplus \mathcal{O}(-1) \oplus \mathcal{O}(-2) \rightarrow \mathbb{P}^{1}$, a complex four-fold, which is consistent with the four-fold representation obtained for the $D_{4}$ singularity above.

However, the mirror map on $D_{1}$ and that on $X_{1}$ are not the same. What does this mean? Can we hope that the mirror map is somehow getting simpler with increased dimension, as on the $X_{-1} \cong A_{1}$ example?

The answer, as well as the means of producing rational Yukawa couplings, lies in specializing the torus weights to the equivariantly Calabi-Yau setting $\lambda_{1}=-\lambda_{2}=\lambda$. Now consider the differential operator which annihilates the $I$ function of the $D_{1}$ singularity:

$$
\mathcal{D}_{D_{1}}=\theta^{2}(\theta+\lambda)-q(-\theta-\lambda)(-2 \theta-\lambda)(-2 \theta-\lambda-\hbar)
$$

This operator can be factorized as

$$
\mathcal{D}_{D_{1}}=\left(\theta^{2}+q(-2 \theta-\lambda)(-2 \theta-\lambda-\hbar)\right)(\theta+\lambda)
$$

Then equation (3.20) asserts the equivalence of the operators $\theta^{2}+q(-2 \theta-\lambda)$ $(-2 \theta-\lambda-\hbar)$ and $\theta^{2}+q(\theta+\lambda)^{2}$, from which we expect that we can also reproduce equivalent Gromov-Witten invariants by use of the operator

$$
\begin{aligned}
\mathcal{D}^{\prime} & =\left(\theta^{2}+q(\theta+\lambda)^{2}\right)(\theta+\lambda) \\
& =\theta^{2}(\theta+\lambda)-q(-\theta-\lambda)^{3} .
\end{aligned}
$$

This last form of the $\mathcal{D}^{\prime}$ operator can be derived from the toric data

$$
\left(\begin{array}{llllll}
1 & 1 & 1 & -1 & -1 & -1 \\
0 & 0 & \lambda & -\lambda & -\lambda & -\lambda
\end{array}\right)
$$

and corresponds to the total space $\mathcal{O}(1) \oplus \mathcal{O}(-1) \oplus \mathcal{O}(-1) \oplus \mathcal{O}(-1) \rightarrow \mathbb{P}^{1}$ with a torus action $(\lambda,-\lambda,-\lambda,-\lambda)$ on the bundle. Again, we may directly 
compute to verify that indeed the equivariant Gromov-Witten invariants corresponding to this toric data agree with those of $X_{1}$ with the antidiagonal action.

Now we ask what we have gained through these geometric manipulations. We consider the $I$ function which generates the solution space of $\mathcal{D}^{\prime} f=0$ :

$$
I^{\prime}=e^{p \log q / \hbar} \sum_{d \geq 0} \frac{\prod_{i=-d+1}^{0}(-p-\lambda+m \hbar)^{3}}{\prod_{i=1}^{d}(p+\lambda+m \hbar) \prod_{i=1}^{d}(p+m \hbar)^{2}} q^{d}
$$

We run $I^{\prime}$ through the same procedure described in Section 3.1: expand $I^{\prime}$ about $\lambda=\infty$ and then perform Birkhoff factorization of the result. After this, we find the following incredible result:

$$
J^{\prime}=1+\frac{p(\log q+3 \log (1+q))+\lambda \log (1+q)}{\hbar}+\cdots
$$

In other words, the mirror map has taken on the nearly trivial form $t=\log q+3 \log (1+q)$, and moreover, the equivariant mirror map (the coefficient of $\lambda$ in equation (3.43)) is just a multiple of the regular mirror map.

\subsection{Natural rational $B$ model Yukawa couplings}

We now argue that the natural choice of the triple intersection number of the curve, from the perspective of the $B$ model calculation, should be $\frac{1}{k(k+2)}$. This will give the simple form of the Picard-Fuchs equation and $B$ model Yukawa coupling of Conjecture 1. Moreover, this agrees with the predictions of $[2]$.

We consider the prepotential of $X_{k}$ with antidiagonal action and arbitrary triple intersection number $\langle C, C, C\rangle$. Using the expansion of [14], this is

$$
\mathcal{F}_{k}=\langle C, C, C\rangle \frac{t^{3}}{3 !}-\sum_{d=1}^{\infty} \frac{(-1)^{k d}\left((k+1)^{2} d-1\right) !}{d ! d^{2}\left(\left((k+1)^{2}-1\right) d\right) !} e^{t d} .
$$

The key to choosing the right intersection number now actually lies in looking at the second derivative of this function. An easy computation shows that

$$
\frac{\partial^{2} \mathcal{F}_{k}}{\partial t_{k}^{2}}=\langle C, C, C\rangle \log \left(q\left(1+(-1)^{k+1} q\right)^{k(k+2)}\right)+\log \left(1+(-1)^{k+1} q\right) .
$$


Then by setting the value

$$
\langle C, C, C\rangle=\frac{-1}{k(k+2)},
$$

we immediately find the remarkably simple relation

$$
\frac{\partial^{2} \mathcal{F}_{k}}{\partial t_{k}^{2}}=\frac{-1}{k(k+2)} \log q
$$

Rather than being merely the choice of intersection number which gives the simplest expression of the above function, there are several other reasons to expect that this is the correct choice (besides the fact that it was predicted to be so in [2]). The first is by examining the form of the Picard-Fuchs equation which results from the above Yukawa coupling. The Picard-Fuchs equation describing mirror symmetry will be given by

$$
\begin{aligned}
\partial_{t_{k}}\left(\frac{1}{Y_{t_{k} t_{k} t_{k}}}\right) \partial_{t_{k}}^{2} & =\theta k(k+2) q \frac{d t_{k}}{d q}\left(q \frac{d t_{k}}{d q}\right)^{-1} \theta\left(q \frac{d t_{k}}{d q}\right)^{-1} \theta \\
& =\theta^{2}\left(q \frac{d t_{k}}{d q}\right)^{-1} \theta
\end{aligned}
$$

In other words, only the information of the mirror map is required to compute all Gromov-Witten invariants of $X_{k}$. Said differently, mirror symmetry is completely characterized by the integer $k$ (since the mirror map is).

Another reason the above choice of intersection number is the most natural can be seen from the genus 1 function in the next section. First, note that by using the above expression for $\partial^{2} \mathcal{F} / \partial t^{2}$, we have the $B$ model Yukawa coupling, as given in Conjecture 1:

$$
Y_{q q q}=\frac{\partial^{3} \mathcal{F}}{\partial t^{3}}\left(q \frac{d t_{k}}{d q}\right)^{3}=\frac{-1}{k(k+2)}\left(q \frac{d t_{k}}{d q}\right)^{2} .
$$

As is evident from the expansions of the next section, there are two components of the discriminant locus, given by the numerator and denomenator of the mirror map, respectively. Now, if we make any other choice of triple intersection number, what happens is that the $B$ model Yukawa coupling will contain an extra polynomial factor. From the perspective of period integrals, this suggests that there will be an extra component of the discriminant locus, which one would reasonably expect to appear in the genus 1 function. However, as this extra component does not appear, it is natural that the Yukawa coupling should have the same singular points as the mirror map, thus lending support to our choice of intersection number. 
Finally, we can also use the formula proposed in [8] for the computation of triple intersection numbers. Adapted to the present case, this reads

$$
\begin{aligned}
\langle C, C, C\rangle & =\int_{\mathbb{P}^{1}} \frac{J^{3}}{e(\mathcal{O}(k) \oplus \mathcal{O}(-2-k))} \\
& =\int_{\mathbb{P}^{1}} \frac{J^{3}}{J^{2} k(-2-k)}=-\frac{1}{k(2+k)} \int_{\mathbb{P}^{1}} J
\end{aligned}
$$

where $J$ is the Kähler class satisfying $\int_{\mathbb{P}^{1}} J=1$ and $e()$ denotes the Euler class. Hence we obtain the intersection number claimed.

\subsection{Genus one}

With such a simple form for the Yukawa coupling and mirror map at hand, it is natural to suppose that we also have an elementary form for the genus 1 Gromov-Witten potential of $X_{k}$ on the $B$ model, which we denote by $G_{k}$. In fact, our interest in doing the genus 1 computation was the original motivation behind this project.

The $A$ model function can be worked out by the topological vertex [1]. We are grateful to Konishi for providing us with a program for the vertex calculation. Then, we need only compare the general form of the $B$ model genus 1 amplitude to see if it agrees with the $A$ model answer. Recall [3] that the $B$ model function $G_{k}$ has the general structure

$$
G_{k}=\log \left(q^{a} \prod_{i} \Delta_{i}^{b_{i}} J_{k}\right)
$$

where $\Delta_{i}$ is and irreducible component of the discriminant locus, $J_{k}=$ $d \log q / d t_{k}$ is the Jacobian and $a, b$ are rational numbers. We again specialize to the case $X_{1}$ for clarity. Then we have $J_{1}=(1+q) /(1+4 q)$, and since this is the derivative of the mirror map, the singular points of $J_{1}$ define the discriminant locus:

$$
\Delta_{1}=1+q, \quad \Delta_{2}=1+4 q .
$$

Then a simple calculation verifies that $G_{1}$ is given as

$$
G_{1}(q)=\frac{11}{24} \log (1+4 q)-\frac{1}{4} \log (1+q)+\frac{1}{2} \log \left(J_{1}\right)
$$

By substituting the inverse mirror map into $G_{1}$, we find the expansion

$$
G_{1}(t)=\frac{1}{12} e^{t}-\frac{1}{24} e^{2 t}-\frac{29}{36} e^{3 t}+\frac{499}{48} e^{4} t-\frac{517}{5} e^{5 t}+\cdots
$$


In exactly the same way, we find $G_{2}$ :

$$
G_{2}=\frac{11}{24} \log (1-9 q)-\frac{1}{24} \log (1-q)+\frac{1}{2} \log \left(J_{2}\right)
$$

where now $J_{2}=(1-q) /(1-9 q)$. This has expansion

$$
G_{2}(t)=-\frac{1}{12} e^{t}+\frac{19}{24} e^{2 t}+\frac{899}{36} e^{3 t}+\frac{27259}{48} e^{4 t}+\frac{733289}{60} e^{5 t}+\cdots
$$

By checking a few more cases we have formulated Conjecture 2 .

\subsection{A word about the diagonal action}

We now briefly consider equivariant mirror symmetry for $X_{k}$ with the diagonal torus action $\lambda_{1}=\lambda_{2}$. We will see that this is very likely the same calculation as the projective bundle $Y_{k}=\mathbb{P}(\mathcal{O} \oplus \mathcal{O}(k) \oplus \mathcal{O}(-2-k)) \rightarrow \mathbb{P}^{1}$. Unfortunately, the techniques of the earlier sections do not give any simple form for the mirror map or genus 1 expansion, but as $Y_{k}$ is not Calabi-Yau, this is not entirely unexpected.

We see some hint of the correspondence already between the spaces $X_{-1}$ and $Y_{-1}$. We have the expansion for the equivariant $I$ function on $X_{-1}$ :

$$
\begin{aligned}
I_{X_{-1}} & =e^{p \log q / \hbar} \sum_{d \geq 0} \frac{\prod_{m=-d+1}^{0}(-p+m \hbar+\lambda)}{\prod_{m=1}^{d}(p+m \hbar)} q^{d} \\
& =1+\frac{p \log q}{\hbar}+\frac{\lambda^{2} L i_{2}(q)-2 p \lambda L i_{2}(q)}{\hbar^{2}}+\cdots
\end{aligned}
$$

and that on $Y_{-1}$ :

$$
\begin{aligned}
& I_{Y_{-1}} \\
& =e^{\left(p_{1} \log q_{1}+p_{2} \log q_{2}\right) / \hbar} \sum_{d \geq 0} \frac{\prod_{m=-\infty}^{0}\left(-p_{1}+p_{2}+m \hbar\right)}{\begin{array}{l}
\prod_{m=1}^{d_{1}}\left(p_{1}+m \hbar\right)^{2} \prod_{m=-\infty}^{-d_{1}+d_{2}} \\
\left(-p_{1}+p_{2}+m \hbar\right) \prod_{m=1}^{d_{2}}\left(p_{2}+m \hbar\right)
\end{array}} q_{1}^{d_{1}} q_{2}^{d_{2}} \\
& =1+\frac{p_{1} \log q_{1}+p_{2} \log q_{2}}{\hbar} \\
& \quad+\frac{p_{2}^{2} L i_{2}\left(q_{1}\right)-2 p_{1} p_{2} L i_{2}\left(q_{1}\right)+\log q_{1} \log q_{2}+\left(\log q_{2}\right)^{2} / 2}{\hbar^{2}}+\cdots
\end{aligned}
$$

The expansions disagree at higher order in $1 / \hbar$, but one might simply attribute this to the fact that $I_{Y_{-1}}$ is considered as a cohomology valued 
hypergeometric series, taking values in the cohomology ring

$$
\frac{\mathbb{C}\left[p_{1}, p_{2}\right]}{\left\langle p_{1}^{2}, p_{2}\left(p_{1}-p_{2}\right)^{2}\right\rangle},
$$

while the coefficients of $I_{X_{-1}}$ are only subjected to the relation $p^{2}=0$. This argument is strengthened if one looks at the next example:

$$
\begin{aligned}
& I_{X_{0}}=1+\frac{p(\log q+2 f(q))-\lambda f(q)}{\hbar}+\cdots, \\
& I_{Y_{0}}=1+\frac{p_{1}\left(\log q_{1}+2 f\left(q_{1}\right)\right)+p_{2}\left(\log q_{2}-f\left(q_{1}\right)\right)}{\hbar}+\cdots,
\end{aligned}
$$

where $f(q)=\sum_{n>0} q^{n}(2 n-1) ! /(n !)^{2}$. That is, the mirror maps are the same, and moreover, the instanton expansions agree exactly after inversion of the respective mirror maps.

The more nontrivial statement is that this correspondence holds even across Birkhoff factorization. Namely, if we consider $X_{1}$ with the diagonal torus action $(\lambda, \lambda)$ and carry out Birkhoff factorization on the resulting $I$ function, both the mirror map and the Gromov-Witten invariants turn out to be exactly the same as those of the projective bundle $Y_{1}=$ $\mathbb{P}(\mathcal{O} \oplus \mathcal{O}(1) \oplus \mathcal{O}(-3)) \rightarrow \mathbb{P}^{1}$, where we have performed Birkhoff factorization on the $I$ function for $Y_{1}$.

Unfortunately, we were unable to obtain nice formulas for any of the quantities discussed in this paper on $Y_{k}$. Hence, the analog of Picard-Fuchs equations, etc. remains unclear.

\section{Equivariant mirror symmetry for $X$ with $\operatorname{dim} H_{4}(X)=0$}

We now show that equivariant techniques can be used to effectively compute Gromov-Witten potentials not only for bundles over curves, but in fact for any Calabi-Yau lacking four cycles.

\section{$4.1 \quad A_{2}$}

Consider the toric charge vectors for the standard $A_{2}$ geometry

$$
\left(\begin{array}{cccc}
1 & -2 & 1 & 0 \\
0 & 1 & -2 & 1
\end{array}\right)
$$

This has two curve classes $C_{1}, C_{2}$ corresponding to the rows of the above matrix. Also let $p_{1}, p_{2}$ be Kähler classes satisfying $\int_{C_{i}} p_{j}=\delta_{i j}$. Note that 
for $C_{1}$, the normal bundle direction is given by the second column of the matrix, and for $C_{2}$, the normal bundle corresponds to the third column. As such, $\lambda_{1}$ is the equivariant parameter corresponding to the $C_{1}$ curve, and $\lambda_{1}$ to $C_{2}$.

Now, in order to exhibit equivariant Gromov-Witten invariants on the $A_{1}$ singularity, we simply added an equivariant parameter corresponding to the normal bundle direction:

$$
\left(\begin{array}{lll}
1 & 1 & -2
\end{array}\right) \longrightarrow\left(\begin{array}{ccc}
0 & 0 & \lambda \\
1 & 1 & -2
\end{array}\right)
$$

Hence the most natural equivariant theory we can use to extract GromovWitten invariants is

$$
\left(\begin{array}{cccc}
0 & \lambda_{1} & \lambda_{2} & 0 \\
1 & -2 & 1 & 0 \\
0 & 1 & -2 & 1
\end{array}\right)
$$

The strategy is then simple enough: just use the equivariant $I$ function to compute the mirror map and prepotential. The $I$ function is

$$
I_{A_{2}}=e^{\left(p_{1} \log q_{1}+p_{2} \log q_{2}\right) / \hbar} \sum_{d \geq 0} C\left(d_{1}, d_{2}, \lambda\right) q_{1}^{d_{1}} q_{2}^{d_{2}}
$$

where $C\left(d_{1}, d_{2}, \lambda\right)=$

$$
\begin{gathered}
\prod_{m=-\infty}^{0}\left(-2 p_{1}+p_{2}+\lambda_{1}+m \hbar\right) \prod_{m=-\infty}^{0}\left(p_{1}-2 p_{2}+\lambda_{2}+m \hbar\right) \\
\prod_{m=1}^{d_{1}}\left(p_{1}+m \hbar\right) \prod_{m=-\infty}^{-2 d_{1}+d_{2}}\left(-2 p_{1}+p_{2}+\lambda_{1}+m \hbar\right) \prod_{m=-\infty}^{d_{1}-2 d_{2}} \\
\left(p_{1}-2 p_{2}+\lambda_{2}+m \hbar\right) \prod_{m=1}^{d_{2}}\left(p_{2}+m \hbar\right)
\end{gathered}
$$

Recall that these coefficients are subjected to the cohomology relations $p_{1}^{2}=$ $p_{1} p_{2}=p_{2}^{2}=0$. Then we go through the usual motions of expanding this function in powers of $1 / \hbar$ and inverting the mirror map and equivariant mirror map, which are given by the coefficient of $1 / \hbar$. Let $t_{1}, t_{2}$ be the mirror map, and set $x_{1}=e^{t_{1}}, x_{2}=e^{t_{2}}$. Let $J_{A_{n}}$ be the function obtained by coordinate change of $I_{A_{n}}$ by the mirror map. Then we want to read the instanton information from the coefficient $W$ of $1 / \hbar^{2}$ of $J_{A_{n}}$. There is one minor point one needs to keep in mind when extracting the instanton information: since the $\lambda_{1}$ equivariant parameter corresponds to the curve $C_{1}$, there are certain 'anomalous' terms in $W$ which mix the normal bundle of $C_{1}$ and the curve $C_{2}$. We can cancel these terms by looking at one 
equivariant parameter at a time: if we set $p_{2}=\lambda_{2}=0$, we obtain

$$
\begin{aligned}
\left.W\right|_{\lambda_{2}=0, p_{2}=0}= & \lambda_{1}^{2}\left(L i_{2}\left(x_{1}\right)+L i_{2}\left(x_{1} x_{2}\right)\right)+p_{1} \lambda_{1}\left(2 L i_{2}\left(x_{1}\right)\right. \\
& \left.+L i_{2}\left(x_{1} x_{2}\right)-L i_{2}\left(x_{2}\right)\right) \\
= & \lambda_{1}^{2} \frac{\partial \mathcal{F}}{\partial t_{1}}+p_{1} \lambda_{1}\left(2 \frac{\partial \mathcal{F}}{\partial t_{1}}-\frac{\partial \mathcal{F}}{\partial t_{2}}\right)
\end{aligned}
$$

where

$$
\mathcal{F}=L i_{3}\left(x_{1}\right)+L i_{3}\left(x_{2}\right)+L i_{3}\left(x_{1} x_{2}\right) .
$$

Note that this is just as one would expect from local mirror symmetry calculations, since the coefficient of $p_{1} \lambda_{1}$ of equation (4.67) features a linear combination of prepotential derivatives determined by the second column of the matrix (4.65).

Now that we know the instanton expansion, we can use this to work out mirror symmetry at genus 1 for the $A_{2}$ singularity. Unfortunately, the geometry of the mirror of (4.65) is degenerate, which means we cannot easily extract the discriminant locus from the mirror manifold. Hence, we instead use a $\mathbb{P}^{1}$ fibration over $A_{2}$

$$
\left(\begin{array}{cccccc}
1 & 1 & -2 & 0 & 0 & 0 \\
0 & 0 & 1 & -2 & 1 & 0 \\
0 & 0 & 0 & 1 & -2 & 1
\end{array}\right)
$$

We then compute the discriminant locus from the mirror manifold, and then take the limit as the first curve disappears. The result is

$$
\begin{aligned}
\Delta= & 1-8 q_{1}-8 q_{2}+68 q_{1} q_{2}+16 q_{1}^{2}+16 q_{2}^{2}-144 q_{1} q_{2}^{2}-144 q_{1}^{2} q_{2}+270 q_{1}^{2} q_{2}^{2} \\
& +216 q_{1}^{3} q_{2}^{2}+216 q_{1}^{2} q_{2}^{3}-972 q_{1}^{3} q_{2}^{3}+729 q_{1}^{4} q_{2}^{4},
\end{aligned}
$$

from which we can immediately exhibit genus 1 mirror symmetry:

$$
\begin{aligned}
G_{A_{2}} & =\frac{t_{1}}{12}+\frac{t_{2}}{12}-\frac{1}{12} \log \left(\left(1-e^{t_{1}}\right)\left(1-e^{t_{2}}\right)\left(1-e^{t_{1}+t_{2}}\right)\right) \\
& =\log \left(q_{1}^{1 / 12} q_{2}^{1 / 12} \Delta^{-7 / 24}\left(\frac{\partial \log q}{\partial t}\right)^{1 / 2}\right) .
\end{aligned}
$$

$\partial \log q / \partial t$ is the Jacobian of the mirror map. The coefficient of $-7 / 24$ is also the same for the $A_{1}$ singularity, suggesting that this behavior may be universal for $A_{n}$ singularities.

\subsection{The trivalent $(-1,-1)$ curve}

Since this example is closely related to the above, we give only the briefest discussion, merely indicating the points at which this differs from $A_{n}$. The 
geometry we are considering has three curves with normal bundle $\mathcal{O}(-1) \oplus$ $\mathcal{O}(-1)$ and all three curves intersect at a single point. The equivariant theory we use is thus

$$
\left(\begin{array}{cccccc}
0 & 0 & 0 & \lambda_{1} & \lambda_{2} & \lambda_{3} \\
1 & 0 & 0 & 1 & -1 & -1 \\
0 & 1 & 0 & -1 & 1 & -1 \\
0 & 0 & 1 & -1 & -1 & 1
\end{array}\right)
$$

since the last three columns correspond to noncompact divisors. Then, as above, we can use the equivariant $I$ function to work out mirror symmetry. Let $W$ be the coefficient of $1 / \hbar^{2}$ of the mirror map transformed $J$ function. Then we restrict to the curve $C_{1}$ corresponding to the second row of the above matrix by setting $\lambda_{1}=p_{2}=p_{3}=0$. If we choose the diagonal action, for which $\lambda_{2}=\lambda_{3}=\lambda$,

$$
\left.W\right|_{\lambda_{1}=p_{2}=p_{3}=0, \lambda_{2}=\lambda_{3}=\lambda}=\lambda^{2} \frac{\partial \mathcal{F}_{1}}{\partial t_{1}}+p_{1} \lambda\left(2 \frac{\partial \mathcal{F}_{1}}{\partial t_{1}}-\frac{\partial \mathcal{F}_{1}}{\partial t_{2}}-\frac{\partial \mathcal{F}_{1}}{\partial t_{3}}\right)
$$

and for the antidiagonal action,

$$
\left.W\right|_{\lambda_{1}=p_{2}=p_{3}=0, \lambda_{2}=-\lambda_{3}=\lambda}=\lambda^{2} \frac{\partial \mathcal{F}_{2}}{\partial t_{1}}+p_{1} \lambda\left(\frac{\partial \mathcal{F}_{2}}{\partial t_{3}}-\frac{\partial \mathcal{F}_{2}}{\partial t_{2}}\right)
$$

where

$$
\begin{aligned}
\mathcal{F}_{k}= & \sum_{i=1}^{3} L i_{3}\left(x_{i}\right)+L i_{3}\left(x_{1} x_{2} x_{3}\right)+(-1)^{k+1}\left(L i_{3}\left(x_{1} x_{2}\right)\right. \\
& \left.+L i_{3}\left(x_{1} x_{3}\right)+L i_{3}\left(x_{2} x_{3}\right)\right) .
\end{aligned}
$$

In other words, the use of the diagonal versus antidiagonal action changes the relative sign between $(-1,-1)$ curves and $(0,-2)$ curves. Naturally, this means that neither sign choice is preferred equivariantly, and indeed this continues to hold true at genus 1 .

\section{Conclusion}

In this paper, we have uncovered a surprisingly simple structure underlying mirror symmetry in genus 0 and 1 on $X_{k}=\mathcal{O}(k) \oplus \mathcal{O}(-2-k) \rightarrow \mathbb{P}^{1}$ with antidiagonal action. Although one would expect some simplification of the calculation using the factorization of the bundle as described in Proposition 1, the miraculous appearance of a rational Yukawa coupling points toward some deeper structure behind the problem. 
There are several possible directions for future work. One obvious problem is the extension of these results to higher genus on the $B$ model, which involves the computation of the holomorphic anomaly at each genus. One might also consider whether there is some nice form for the $B$ model computation on $\mathbb{P}(\mathcal{O} \oplus \mathcal{O}(k) \oplus \mathcal{O}(-2-k))$, or equivalently $X_{k}$ with the diagonal action. We were able to derive rational $B$ model couplings for $\mathbb{P}(\mathcal{O} \oplus \mathcal{O} \oplus$ $\mathcal{O}(-2)$ ), but the complexity of the mirror map for $k \geq 1$ poses a major obstacle. Finally, one could also include open strings into the computation. We expect to address these issues in future work.

\section{Acknowledgments}

The authors would like to thank A. Givental, H. Iritani, S. Hosono, M. Marino, K. Saito and A. Takahashi for helpful discussions. We also thank Y. Konishi for providing us with a computer program for the topological vertex calculation. We also thank N. Caporaso, L. Griguolo, M. Marino, S. Pasquetti and D. Seminara for drawing our attention to the content of [14].

\section{References}

[1] M. Aganagic, A. Klemm, M. Marino and C. Vafa, The topological vertex, Commun. Math. Phys. 254 (2005), 425-478, hep-th/0512227.

[2] M. Aganagic, H. Ooguri, N. Saulina and C. Vafa, Black holes, q-deformed 2d Yang-Mills, and non-perturbative topological strings, Nucl. Phys. B715 (2005), 304-348, hep-th/0411280.

[3] M. Bershadsky, S. Cecotti, H. Ooguri and C. Vafa, Holomorphic anomalies in topological field theories, Nucl. Phys. B405 (1993), 279-304, hep-th/9302103.

[4] J. Bryan and R. Pandharipande, The local Gromov-Witten theory of curves, math.AG/0411037.

[5] T. Coates and A. Givental, Quantum Riemann-Roch, Lefschetz and Serre, math.AG/0110142.

[6] F. Cachazo, S. Katz and C. Vafa, Geometric transitions and $N=1$ Quiver theories, hep-th/0108120.

[7] B. Forbes and M. Jinzenji, Extending the Picard-Fuchs system of local mirror symmetry, J. Math. Phys. 46 (2005), 082302, hep-th/0503098.

[8] B. Forbes and M. Jinzenji, Prepotentials for local mirror symmetry via Calabi-Yau four-folds, JHEP 0603(2006), 061, hep-th/0511005. 
[9] B. Forbes and M. Jinzenji, J functions, non nef toric varieties and equivariant local mirror symmetry of curves, math.AG/0603728.

[10] A. Givental, Simple singularities and integrable hierarchies, In the breadth of symplectic and Poisson geometry, 173-201, Progr. Math., 232, Birkhäuser Boston, Boston, MA, 2005, math.AG/0307176.

[11] M. Jinzenji and M. Naka, unpublished.

[12] S. Katz, Genus zero Gopakumar-Vafa invariants of contractible curves, math.AG/0601193.

[13] S. Katz, P. Mayr and C. Vafa, Mirror symmetry and exact solution of $4 D N=2$ gauge theories I, Adv. Theor. Math. Phys. 1 (1998), 53-114, hep-th/9706110.

[14] N. Caporaso, L. Griguolo, M. Marino, S. Pasquetti and D. Seminara, Phase transitions, double-scaling limit, and topological strings, hep-th/0606120. 
